\title{
Stephan's Quintet: The X-ray anatomy of a multiple galaxy collision
}

\author{
G. Trinchieri ${ }^{1}$, J. Sulentic ${ }^{2}$, D. Breitschwerdt ${ }^{3}$, and W. Pietsch ${ }^{3}$ \\ 1 INAF-Osservatorio Astronomico di Brera, via Brera 28, 20121 Milano Italy \\ 2 Physics \& Astronomy, University of Alabama, USA \\ 3 Max-Planck-Institut für extraterrestrische Physik, Giessenbachstraße, 85740 Garching, Germany
}

Received 10 October 2002 / Accepted 17 January 2003

\begin{abstract}
Chandra observations of the compact galaxy group known as Stephan's Quintet (SQ) are presented. The major morphological features that were discovered with the ROSAT HRI are now imaged with higher resolution and S/N. The large scale shock $(1.5, \sim 40 \mathrm{kpc}$ if at $85 \mathrm{Mpc})$ is resolved into a narrow NS feature embedded in more extended diffuse emission $\left(D \geq 3^{\prime}\right)$. The NS structure is somewhat clumpy, more sharply bounded on the $\mathrm{W}$ side and prominent only in the soft band (energies below $\sim 2 \mathrm{keV}$ ). Its observational properties are best explained as a shock produced by a high velocity encounter between NGC 7318b, a "new intruder", and the intergalactic medium in SQ. The shock conditions near the high speed intruder suggest that a bow shock is propagating into a pre-existing $\mathrm{H}_{\mathrm{I}}$ cloud and heating the gas to a temperature of $0.5 \mathrm{keV}$. The low temperature in the shock is a problem unless we postulate an oblique shock. One member, NGC 7319, hosts a Seyfert 2 nucleus, with an intrinsic luminosity $L_{\mathrm{X}}=10^{43} \mathrm{erg} \mathrm{s}^{-1}$, embedded in a region of more diffuse emission with $10^{\prime}$ radius extent. The nuclear spectrum can be modeled with a strongly absorbed power-law typical of this class of sources. Several additional compact sources are detected including three in foreground NGC 7320. Some of these sources are very luminous and could be related to the ultraluminous $\mathrm{X}$-ray sources found in nearby galaxies.
\end{abstract}

Key words. ISM: general - X-rays: galaxies: clusters - galaxies: ISM - X-rays: ISM

\section{Introduction}

Stephan's Quintet (HCG92, Hickson 1982, hereafter SQ) is the most studied example of the compact group phenomenon. It is composed of six galaxies (Sulentic et al. 2001, S01 hereafter) including a core of three (NGC 7317, NGC 7318a and NGC 7319) with essentially zero velocity dispersion and an unrelated foreground object (NGC 7320). Multiwavelength observations of SQ give strong evidence of multiple episodes of past and recent (current) interaction, most likely caused by acquisition of new members/passage of intruders from the associated larger scale galaxy population near SQ. Both NGC 7320c and NGC 7319 show spiral morphology without detectable $\mathrm{H}_{\mathrm{I}}$ while the other two core members are ellipticals. The last and presumably ongoing event involves the collision of the gas-rich spiral NGC 7318b with the debris field produced by past interactions. About half of that galaxy's ISM has been stripped/shocked. SQ is certainly the best local example of a compact group caught in flagrante delicto with multiple manifestations of interaction events. It is this kind of event that presumably accounts for general compact group characteristics including $\mathrm{H}_{\mathrm{I}}$ deficiency, quenched star formation and an excess

Send offprint requests to: G. Trinchieri, e-mail: ginevra@brera.mi .astro.it of early-type members (e.g. Hickson \& Rood 1988; Sulentic \& de Mello Rabaca 1993; Verdes-Montenegro et al. 2001; Vilchez \& Iglesias-Paramo 1998). SQ is thus an ideal laboratory for studying interactions per se, as well as, an excellent local ana$\log$ to the processes thought to be much more common at high redshift.

We present CHANDRA observations of SQ where we confirm the complex nature of the X-ray emission already reported (Pietsch et al. 1997, S01). Most, if not all, of the basic types of $\mathrm{X}$-ray emission observed from extragalactic sources are found in SQ (AGN, shocks, jets, "normal" galactic emission, diffuse emission), and can be studied in this context.

\section{Basic results of the Chandra data analysis}

A 19.7 ks observation of SQ was obtained with Chandra in July 2000 using the back-illuminated CCD (ACIS-S in imaging configuration). The data were reprocessed using the new calibration files as described in the CIAO documentation. Following the "CIAO Science Threads" at the CXC home page ${ }^{1}$, we verified that the data obtained with standard processing had been properly cleaned (e.g. for high background events). We produced several images with $1^{\prime \prime} \times 1$ " pixel

\footnotetext{
${ }^{1}$ http://asc.harvard. edu/ciao/threads/threads.html
} 


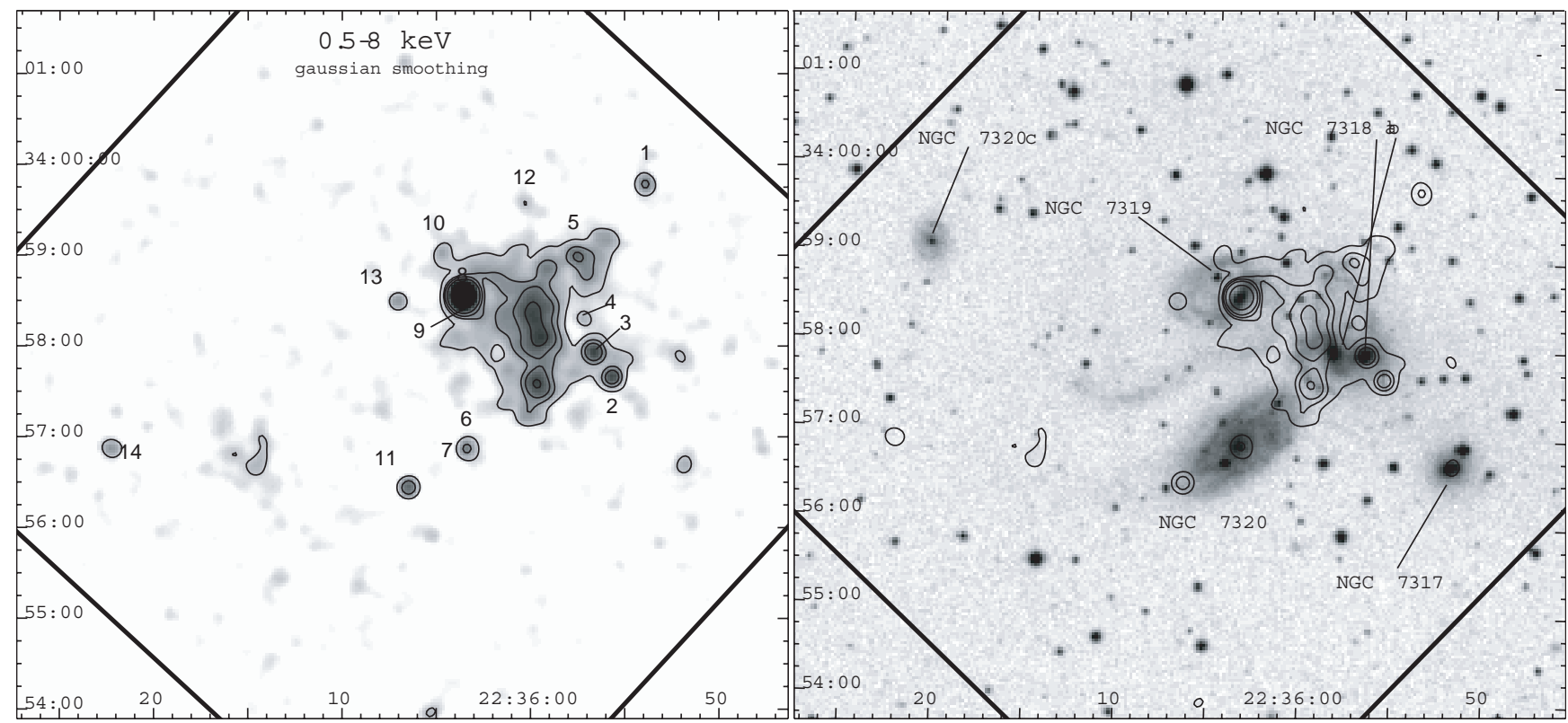

Fig. 1. Left: Full field Chandra image (ACIS-S: back-illuminated chip S3 only) of SQ in the $0.5-8 \mathrm{keV}$ energy range with X-ray contours and source numbers (see Sect. 2.2) superimposed. The map is binned with $1^{\prime \prime} \times 1^{\prime \prime}$ pixels and smoothed with a bidimensional Gaussian filter $\left(\sigma=4^{\prime \prime}\right)$. Right: X-ray contours superimposed on the blue DSS2 image of the field. Galaxies are identified, and the ACIS-S field of view is indicated.

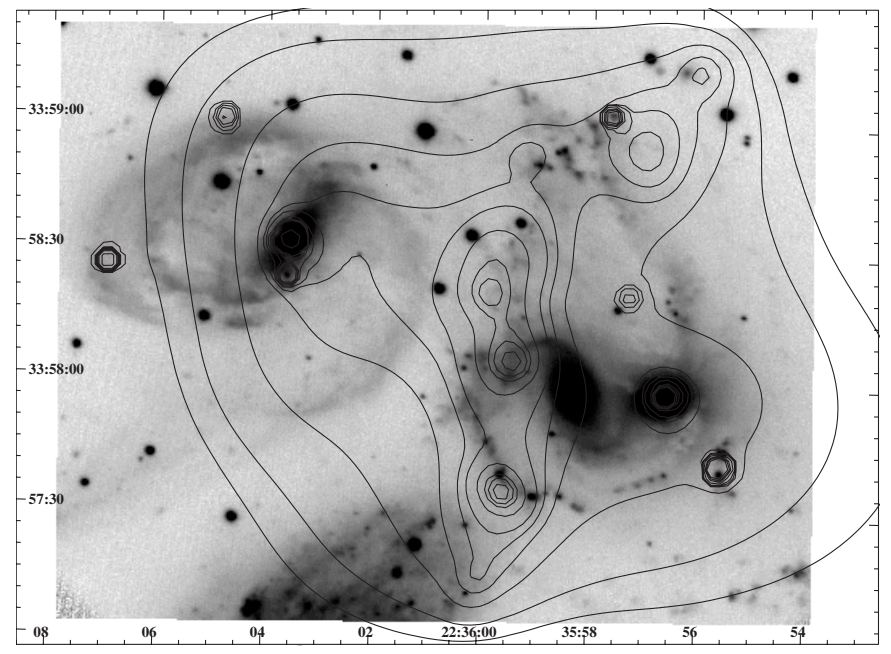

Fig. 2. A zoomed X-ray contour map $(0.5-3 \mathrm{keV})$ of the main concentration of X-ray photons. Contours are superimposed on a CFHT $B$-band image of the field. The X-ray data are smoothed with an adaptive filter and $2.5 \sigma$ as the lowest significance of the signal within the kernel.

resolution, covering different portions of the field and in different energy bands. We applied a Gaussian filter or an adaptive smoothing filter using the csmooth routine in the CIAO package (FFT method, and using $2.5 \sigma$ as the minimal significance of the signal within the kernel). The left panel of Figure 1 presents an X-ray image of the entire back-illuminated CCD chip (S3) with X-ray contours superimposed. The right panel shows X-ray contours superposed on an optical $B$-band image from the DSS2. Considerable extended emission is concentrated within the core of the compact group. Additional sources are associated with the galaxies (members of SQ and the foreground galaxy NGC 7320) or, in some cases, may be unrelated background sources (see Table 1).

Figure 2 presents a closer look at the X-ray emission most unambiguously associated with SQ. The soft $(0.5-3.0 \mathrm{keV})$ $\mathrm{X}$-ray contours are shown overlayed on an average of CFHT $B$-band images kindly provided by C. Mendes de Oliveira (see Plana et al. 1999; Mendes de Oliveira et al. 2001; S01 for discussion of the images). The complex X-ray emission that was detected in previous ROSAT observations (Pietsch et al. 1997; S01) is clearly resolved into two main components almost certainly associated with SQ: 1) complex clumpy and extended emission centered on a radio continuum/optical emission-line emitting shock zone and 2) emission from the Seyfert nucleus in NGC 7319. Additional sources detected do not always have obvious optical counterparts, although they appear to be associated with the galaxies in several cases (the central region of NGC 7318a, and associations in Table 1).

Figure 3 shows Chandra images in different energy ranges. Comparison of the images shows that the extended X-ray emission is found only below $\sim 2 \mathrm{keV}$, and more compact sources coincident with the Seyfert 2 nucleus of NGC 7319 and an unresolved source SW of the NGC 7318a nucleus and apparently coincident with one of the new intruder emission regions (\#14 in Fig. 7 of S01) are prominent at higher energies.

\subsection{Distribution of extended $X$-ray emission}

Diffuse emission in SQ extends from NE of the Seyfert 2 nucleus of NGC 7319 to SW of the nucleus of NGC 7318a. Elongated clumpy NS structure lies near the center of more diffuse emission. We derived azimuthally averaged radial profiles of the extended emission in several energy bands and in several ranges of position angle. The goal of the radial profile analysis 


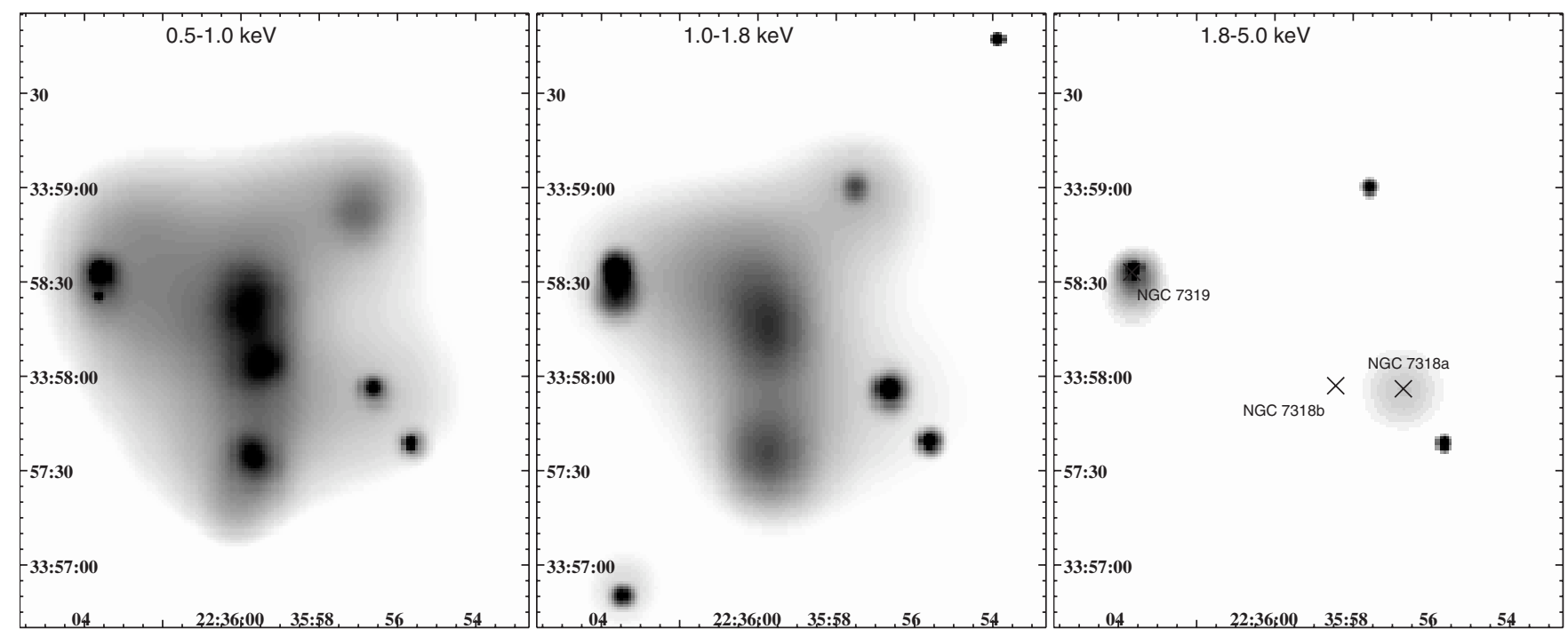

Fig. 3. Smoothed images of the ACIS-S data in SQ, in different energy bands. An adaptive filtering technique (FFT convolution method) is applied to the data. Crosses in the rightmost panel indicate the galaxy's centers.
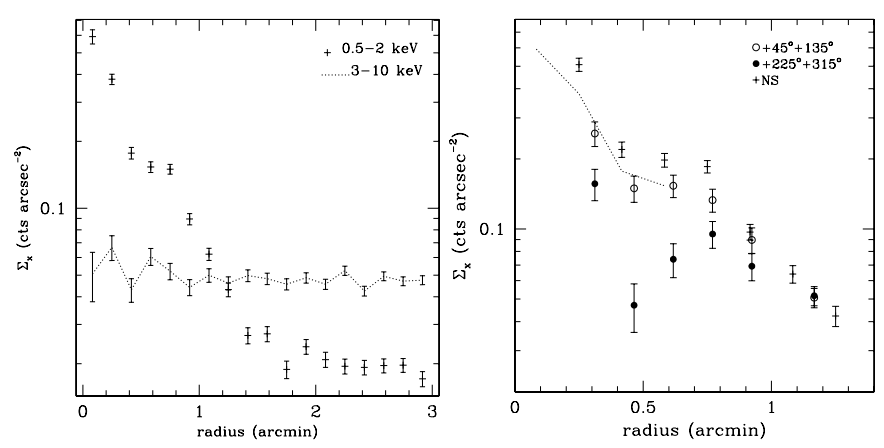

Fig. 4. Left: Azimuthally averaged radial profiles of the total emission in the 0.5-2.0 and 3.0-10.0 keV energy ranges. Right: Radial profile of the total emission in different azimuthal quadrants, as indicated (0.5$2 \mathrm{keV}$ band). The $\pm 45^{\circ}$ azimuthal profiles along the $\mathrm{N}$ and $\mathrm{S}$ directions are very similar and have been averaged together. Only the radial range $0.2-1.5$ is shown because the profiles are the same beyond that range. The $360^{\circ}$ azimuthal average at the center is also shown as a dotted line.

was to determine the extent of diffuse emission for comparison with images at other wavelengths. We centered the radial profiles at RA $=22^{\mathrm{h}} 35^{\mathrm{m}} 59.7^{\mathrm{s}}, \delta=33^{\circ} 58^{\prime} 14.1^{\prime \prime}$ and excluded all discrete sources (Sect. 2.2). Figure 4 shows the azimuthally averaged raw count profiles in two broad energy bands. Extended emission is only detected below $\sim 1.8 \mathrm{keV}$ and out to a radius of $r \sim 2^{\prime}$.

Profiles derived for different azimuthal quadrants indicate that the extent of the emission is similar in all directions but that the intensity distribution is not. Detailed comparison of azimuthal averages is not straightforward because the extended emission is complex. The NS distribution is dominated by the shock. The E profile is similar to the NS distribution although less intense. It shows a smoother decline and a higher intensity level than towards the W. A larger and more abrupt drop in intensity is seen towards the $\mathrm{W}$ followed by an apparent rebound (Fig. 4). The E-W difference is also evident in the EW cut taken

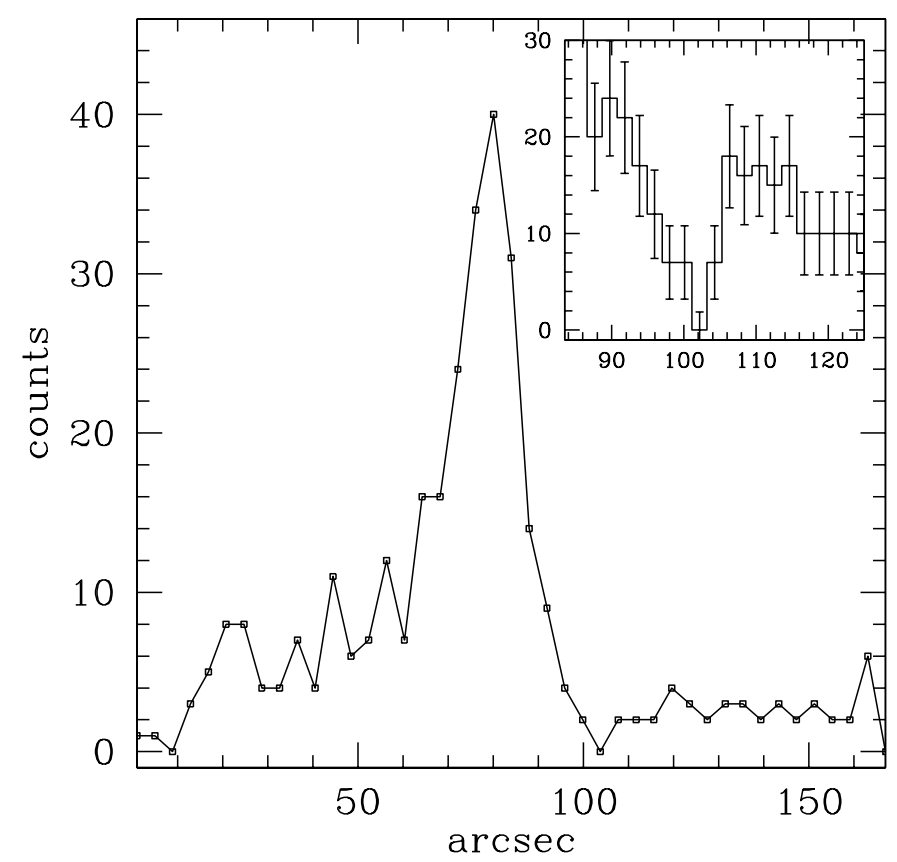

Fig. 5. Total counts in a EW oriented strip centered at $22^{\mathrm{h}} 35^{\mathrm{m}} 59.36^{\mathrm{s}}$, $+33^{\circ} 58^{\prime} 07.2^{\prime \prime}$. The horizontal scale covers $170^{\prime \prime}$, and each point plotted represents the $\left(0.5-2 \mathrm{keV}\right.$ band) counts in bins of size $\sim 4^{\prime \prime} \times 12^{\prime \prime}$. The inset shows an enlargement of the region around $r=100^{\prime \prime}$ corresponding to the minimum intensity, and relative error bars, which was obtained in a much larger area, so that each step represents the counts in $\sim 2^{\prime \prime} \times 62$ ". 5 regions. Counts associated with detected sources are not included.

across the middle intensity peak of the NS feature (Fig. 5). This plot indicates a sharp decrease to a very low count level in a narrow and elongated region $\left(\sim 8^{\prime \prime} \times 1^{\prime}\right)$ along the $\mathrm{W}$ edge of the NS feature. The emission increases again $\mathrm{W}$ of this minimum and extends outwards for an additional $50^{\prime \prime}-70^{\prime \prime}$. The low intensity strip is not aligned with the pixels on the CCD chip which leads us to conclude that it is not an instrumental artifact. 
An additional weak extended X-ray component is seen near $\mathrm{RA}=22^{\mathrm{h}} 36^{\mathrm{m}} 15^{\mathrm{s}}, \delta=+33^{\circ} 56^{\prime} 30^{\prime \prime}$. This $15^{\prime \prime}$ radius region $(\sim 2-3 \sigma$ source) coincides with structure in the older tidal tails (Arp \& Lorre 1976; S01). However, since several optical condensations are found in this same region we cannot rule out a line of sight coincidence with background sources. We will explore this feature in more detail with recently obtained XMMNewton data (Trinchieri et al., in prep).

\subsection{Discrete $X$-ray sources}

A considerable number of discrete sources is found in and near SQ. Some are resolved and other are not, while several show associated (i.e. concentric) extended emission. Table 1 lists discrete sources with positions, count statistics, fluxes, X-ray luminosities and possible optical counterparts (see Fig. 1). The source list is derived from the wavdetect and celldetect programs that gave virtually identical results. The background is derived locally around each source and is negligible for sources outside the region where complex emission is detected. Count rates in the total energy band are converted into unabsorbed fluxes and luminosities assuming a constant conversion rate of $1 \mathrm{c} \mathrm{s}^{-1}=9 \times 10^{-12} \mathrm{erg} \mathrm{cm}^{-2} \mathrm{~s}^{-1}$ (corresponding to either a power law with $\Gamma=1.7$, or a bremsstrahlung with $k T=10 \mathrm{keV}$, and line-of-sight absorption), and a distance of 85.6 Mpc for SQ sources, and 12 Mpc for NGC 7320 (see Table 1). Only fluxes are quoted for sources outside or not unambiguously associated with SQ.

\subsection{X-ray spectra for the strongest components}

Given the statistical significance of the Chandra data, we confine our spectral analysis to the extended emission and the central regions of NGC 7319. We used the psextract script in CIAO to create appropriate spectral matrixes (eg. arf and $r m f$ files) for the analysis, which was done using XSPEC. While the script is not appropriate for extended emission, the regions we have selected are small and, consequently, the error introduced by the point-source assumption is negligible (see also discussion in Trinchieri \& Goudfrooij 2002; Hicks et al. 2002). The degradation of the ACIS Quantum efficiency, recently discussed $^{2}$ was considered. However, our data are not affected significantly because the observation was done at early stages in the mission.

\subsubsection{The NS extended feature}

We identified a region along the NS feature that includes the three brightest condensations. The background was obtained from adjacent regions. The data was binned to yield a $S / N \geq 3$ per bin after background subtraction. This feature was only detected in the $0.35-1.75 \mathrm{keV}$ energy range. We adopted a plasma code (MEKAL model in XSPEC) to describe the data which gives best fit parameters $k T \sim 0.53 \mathrm{keV}$ (0.47-0.58 at $90 \%$ confidence for one interesting parameter), low abundance $(0.12-0.30)$ and absorption consistent with the

\footnotetext{
${ }^{2}$ http://asc.harvard.edu/cal/Acis/Cal_prods/qeDeg
}
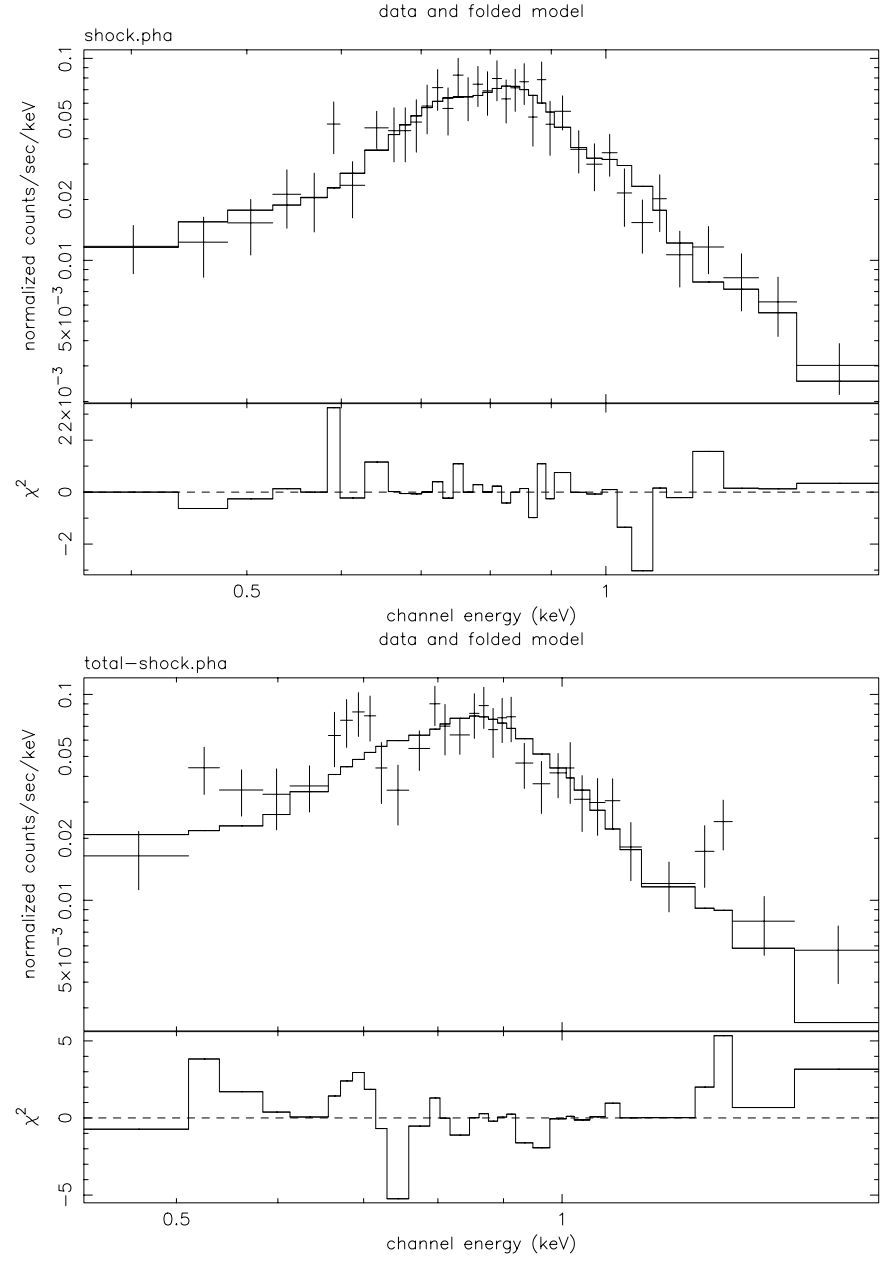

Fig. 6. Spectral distribution and plots of the contributions to $\chi^{2}$ in the extended NS source (TOP) and in the residual surrounding emission (BOTTOM). Error bars on the $y$-axis indicate the statistical uncertainty, on the $x$-axis the width of the energy bin. The model is in both cases a MEKAL with galactic line-of-sight absorption, low ( 20\% cosmic) abundances, and $k T \sim 0.5 \mathrm{keV}$ (top panel) and $k T \sim 0.6 \mathrm{keV}$ (bottom panel); see text.

galactic line of sight value of $N_{\mathrm{H}} \sim 8 \times 10^{20} \mathrm{~cm}^{-2}$ (see Fig. 6). Assuming the best fit model parameters, we derive an unabsorbed $f_{\mathrm{X}}(0.1-2 \mathrm{keV}) \sim 1.7 \times 10^{-13} \mathrm{erg} \mathrm{cm}^{-2} \mathrm{~s}^{-1}$, and $L_{\mathrm{X}} \sim 1.5 \times 10^{41} \mathrm{erg} \mathrm{\textrm {s } ^ { - 1 }}$. Some residuals might be present possibly even coincident with oxygen lines that are not accounted for by the assumed single temperature model. They might be indicative of more complex gas properties (see Fig. 6). We also note that the derived best fit parameters are far from unique: a two temperature component spectrum, with a $0.5 \mathrm{keV}$ MEKAL plasma and solar abundances plus $\sim 5 \mathrm{keV}$ bremsstrahlung component yield an equally good fit to the NS feature. Moreover, a crude examination of the X-ray colors along the NS feature indicates that the three clumps might have different energy distributions, suggesting spectral variations even within this small region.

The lower surface brightness emission around the NS feature (i.e. what we used as background) was also modeled (Fig. 6). We selected a circle of 1.5 radius, excluding discrete sources (e.g. NGC 7318a and NGC 7319) and the NS feature. 
Table 1. Positions, net counts in the broad energy band $(0.3-10 \mathrm{keV})$, fluxes and luminosities $(0.3-10 \mathrm{keV})$ in c.g.s. units of the sources detected in SQ. Net counts are derived in a $1^{\prime \prime}$ radius circle above the local background (except for source \#14, for which $r=3^{\prime \prime}$, and converted into fluxes assuming a conversion factor $4.6 \times 10^{-16}$, corresponding to a Bremsstrahlung model of $10 \mathrm{keV}$ except for the nuclear region in NGC 7319 , where the best fit from the spectral analysis is used.

\begin{tabular}{|c|c|c|c|c|c|c|c|}
\hline CXC name & $\begin{array}{c}\text { Sou } \\
\#\end{array}$ & $\begin{array}{r}\text { RA / Dec } \\
(\mathrm{J} 2000)\end{array}$ & Total & counts & $(0.3-1$ & $\begin{array}{l}\text { luminosity } \\
(0 \mathrm{keV})\end{array}$ & Notes \\
\hline CXOU J223553.9+335946 & 1 & $\begin{array}{l}22: 35: 53.94 \\
33: 59: 46.91\end{array}$ & 24 & $23.8 \pm 4.9$ & $1.1 \times 10^{-14}$ & & $\begin{array}{l}\text { Background? } \\
\text { no optical counterpart }\end{array}$ \\
\hline CXOU J223555.6+335738 & 2 & $\begin{array}{l}22: 35: 55.69 \\
33: 57: 38.82\end{array}$ & 58 & $57.6 \pm 7.6$ & $2.7 \times 10^{-14}$ & & $\begin{array}{l}\text { Associated with } \\
\text { NGC } 7318 b ?\end{array}$ \\
\hline CXOU J223556.6+335756 & 3 & $\begin{array}{l}22: 35: 56.68 \\
33: 57: 56.04\end{array}$ & $\begin{array}{l}27 \\
72\end{array}$ & $\begin{array}{l}24.4 \pm 5.1 \\
64.4 \pm 8.5\end{array}$ & $\begin{array}{l}1.1 \times 10^{-14} \\
3.0 \times 10^{-14}\end{array}$ & $\begin{array}{l}9.7 \times 10^{39} \\
2.7 \times 10^{40}\end{array}$ & $\begin{array}{l}\text { NGC 7318a } 1^{\prime \prime} \text { radius } \\
\text { NGC 7318a } 5^{\prime \prime} \text { radius }\end{array}$ \\
\hline CXOU J223557.3+335818 & 4 & $\begin{array}{l}22: 35: 57.36 \\
33: 58: 18.76\end{array}$ & 8 & $7.4 \pm 2.8$ & $3.4 \times 10^{-15}$ & & $\begin{array}{l}\text { Near NGC } 7318 b \\
\text { emission reg }\end{array}$ \\
\hline CXOU J223557.6+335859 & 5 & $\begin{array}{l}22: 35: 57.61 \\
33: 58: 59.72\end{array}$ & 26 & $25.4 \pm 5.0$ & $1.2 \times 10^{-14}$ & & $\begin{array}{l}\text { Near NGC } 7318 b \\
\text { emission reg }\end{array}$ \\
\hline CXOU J223603.4+335653 & 6 & $\begin{array}{l}22: 36: 03.40 \\
33: 56: 53.74\end{array}$ & 13 & $12.5 \pm 3.6$ & $5.8 \times 10^{-15}$ & $9.9 \times 10^{37}$ & NGC 7320 nucleus? \\
\hline CXOU J223603.4+335650 & 7 & $\begin{array}{l}22: 36: 03.46 \\
33: 56: 50.40\end{array}$ & 15 & $14.6 \pm 4.1$ & $6.7 \times 10^{-15}$ & $1.2 \times 10^{38}$ & $\begin{array}{l}\text { NGC } 7320 \\
\text { S of nucleus }\end{array}$ \\
\hline CXOU J223603.6+335833 & 8 & $\begin{array}{l}22: 36: 03.60 \\
33: 58: 33.12\end{array}$ & 706 & $699 \pm 26.6$ & $\begin{array}{l}\left(1.4 \times 10^{-11 a}\right) \\
\left(2.6 \times 10^{-14 b}\right)\end{array}$ & $\begin{array}{l}\left(1.1 \times 10^{43 a}\right) \\
\left(2.3 \times 10^{40 b}\right)\end{array}$ & $\begin{array}{l}\text { NGC } 7319 \text { - nucleus } \\
\text { NGC } 7319 \text { - extended }\end{array}$ \\
\hline CXOU J223603.6+335825 & 9 & $\begin{array}{l}22: 36: 03.66 \\
33: 58: 25.01\end{array}$ & 36 & $34.7 \pm 6.0$ & $1.6 \times 10^{-14}$ & $1.4 \times 10^{40}$ & $\begin{array}{l}\text { NGC } 7319 \\
\text { S of nucleus }\end{array}$ \\
\hline CXOU J223604.8+335901 & 10 & $\begin{array}{l}22: 36: 04.82 \\
33: 59: 01.19\end{array}$ & 13 & $12.6 \pm 3.6$ & $5.8 \times 10^{-15}$ & $5.1 \times 10^{39}$ & NGC 7319 \\
\hline CXOU J223606.5+335625 & 11 & $\begin{array}{l}22: 36: 06.50 \\
33: 56: 25.83\end{array}$ & 25 & $24.4 \pm 5.0$ & $1.1 \times 10^{-14}$ & $1.9 \times 10^{38}$ & $\begin{array}{l}\text { NGC } 7320 \\
\text { SE edge }\end{array}$ \\
\hline CXOU J223606.7+340106 & 12 & $\begin{array}{l}22: 36: 06.70 \\
34: 01: 06.40\end{array}$ & 8 & $7.8 \pm 2.8$ & $3.6 \times 10^{-15}$ & & $\begin{array}{l}\text { Background? } \\
\text { no optical counterpart }\end{array}$ \\
\hline CXOU J223607.0+335829 & 13 & $\begin{array}{l}22: 36: 07.05 \\
33: 58: 29.52\end{array}$ & 18 & $17.5 \pm 4.2$ & $8.1 \times 10^{-15}$ & $7.2 \times 10^{39}$ & $\begin{array}{l}\text { NGC } 7319 \\
\text { E source }\end{array}$ \\
\hline CXOU J223622.2+335651 & 14 & $\begin{array}{l}22: 36: 22.29 \\
33: 56: 51.70\end{array}$ & 19 & $13.3 \pm 4.7$ & $6.1 \times 10^{-15}$ & & $\begin{array}{l}\text { Background? } \\
\text { no optical counterpart }\end{array}$ \\
\hline
\end{tabular}

\section{NOTES:}

${ }^{a}$ The flux is derived from the spectral analysis and refers to the nuclear component only (see Sect. 2.3). Counts refer to the total emission within $10^{\prime \prime}$ radius.

${ }^{b}$ The flux refers to the extended emission within a $10^{\prime \prime}$ radius around the nuclear source, and is derived from the spectral analysis (see Sect. 2.3).

Assuming a thermal plasma spectrum with low abundances we find $k T=0.61 \mathrm{keV}(0.56-0.65)$. The total unabsorbed flux of this component is $f_{\mathrm{X}}(0.1-2 \mathrm{keV}) \sim 2.8 \times 10^{-13} \mathrm{erg} \mathrm{cm}^{-2} \mathrm{~s}^{-1}$.

We point out that our interpretation of the NS feature and surrounding area in terms of a recent shock would likely imply different spectral characteristics (namely a multi-temperature non-equilibrium spectrum, cf. Breitschwerdt \& Schmutzler 1999) than those derived above under the assumptions of equilibrium conditions. However, the relatively small number of photons in these components does not allow us to consider more sophisticated models, that might be possible with the higher statistics XMM-Newton data.

\subsubsection{The nuclear region of NGC 7319}

We examined the spectral properties of both the central point source $\left(\sim 1^{\prime \prime}\right.$ radius circle) and a larger region $\left(r=10^{\prime \prime}\right)$ inclusive of it. The nuclear X-ray spectrum is complex and requires multiple components. Comparison with the spectral distribution for the larger region indicates an increase in a soft contribution which may be related to a "galactic" component (see Fig. 7).

Since the optical spectrum indicates a Seyfert 2 nucleus, we assume a combination of absorbed and unabsorbed power law components plus a narrow $6.4 \mathrm{keV}$ emission line (see e.g. Della Ceca et al. 1999; Moran et al. 2001; and references therein). The power law index should be the same for both components with the unabsorbed component representing $\sim 1-10 \%$ of the absorbed one. Moran et al. report two different power-law indices for an average X-ray spectrum involving 29 sources although a single value is consistent with their data. Given the limited statistical significance of our data, we model the nuclear source using a single $\Gamma$ for both power-law components (free) plus a Gaussian line at $6.4 \mathrm{keV}$ (at the redshift of NGC 7319). 


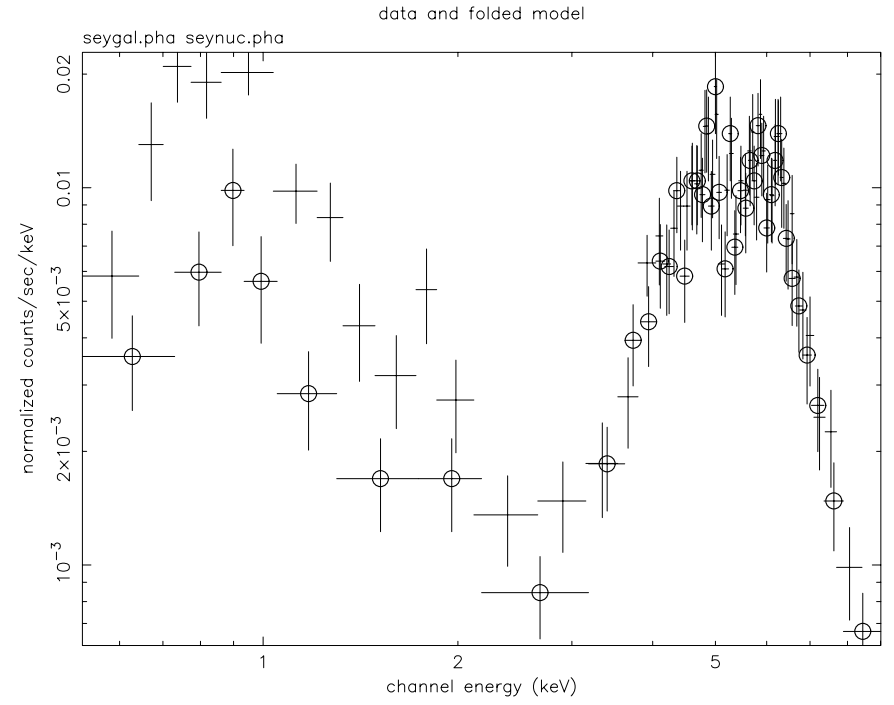

Fig. 7. Comparison of the energy distribution for resolved and unresolved photons from NGC 7319. Error bars on the $y$-axis indicate the statistical uncertainty, on the $x$-axis the width of the energy bin. The larger aperture (10" radius, plus symbol) indicates a significant increase in photons with energies below $2 \mathrm{keV}$ relative to the smaller (1" radius, circle symbols) aperture.

We applied the absorbed+unabsorbed power-law model to both the nuclear and larger regions. Data above $\sim 1.5-2 \mathrm{keV}$ are well described by such a model but excess residual emission is found at lower energies and becomes stronger in the spectrum of the larger region. This motivated us to exclude data below $\sim 2 \mathrm{keV}$ from the fit. Adopting such a "nuclear" model yields best fit $\Gamma \sim 1.7, N_{\mathrm{H}}$ (intrinsic) $=4 \times 10^{23} \mathrm{~cm}^{-2}$. The FeK line is fit with $E=6.4 \mathrm{keV}$ and $E W \sim 110 \mathrm{eV}$. This model gives a good $\chi_{v}^{2} \sim 1$ for 29 degrees of freedom (d.o.f.). Figure 8 shows the spectral data and the contributions to $\chi^{2}$ in individual energy bins relative to the combined plasma + unabsorbed and absorbed power-law model with Gaussian line. A bump is suspected at $E \sim 5.9 \mathrm{keV}$. Although introducing this additional component might improve the fit (the best fit $\chi^{2}$ value reduces by 8 for 3 additional parameters; $f$-test probability $=99.99 \%$ ), there are no known thermal emission lines in this energy range (see Wilms et al. 2001 where a similar feature is suspected in a Seyfert 1 source). The larger aperture data require an additional component at soft energies that can be modeled with either a MEKAL or a raymond plasma code (Raymond \& Smith 1997) with $k T=0.65 \mathrm{keV}$ (MEKAL) or 0.75 (raymond).

The data quality does not allow us to derive meaningful confidence contours, nor does it guarantee that the model is unique. We can however describe the spectral characteristics of the emission from NGC 7319 as due to the superposition of a strong and heavily absorbed nuclear source embedded in more diffuse softer emission. The parameters for the nucleus are consistent with those of a typical Seyfert 2. The intrinsic fluxes derived from the best fit models are: $f_{\mathrm{X}} \sim 4.7 \times 10^{-14} \mathrm{erg} \mathrm{cm}^{-2} \mathrm{~s}^{-1}$ $(0.1-2 \mathrm{keV})$ and $4 \times 10^{-14} \mathrm{erg} \mathrm{cm}^{-2} \mathrm{~s}^{-1}(0.3-10 \mathrm{keV})$ for the extended soft emission; $f_{\mathrm{X}} \sim 1.4 \times 10^{-11} \mathrm{erg} \mathrm{cm}^{-2} \mathrm{~s}^{-1}$ in the entire $0.3-10 \mathrm{keV}$ band and $8.2 \times 10^{-12} \mathrm{erg} \mathrm{cm}^{-2} \mathrm{~s}^{-1}$ in the $2-10 \mathrm{keV}$ band for the Seyfert nucleus, corresponding to
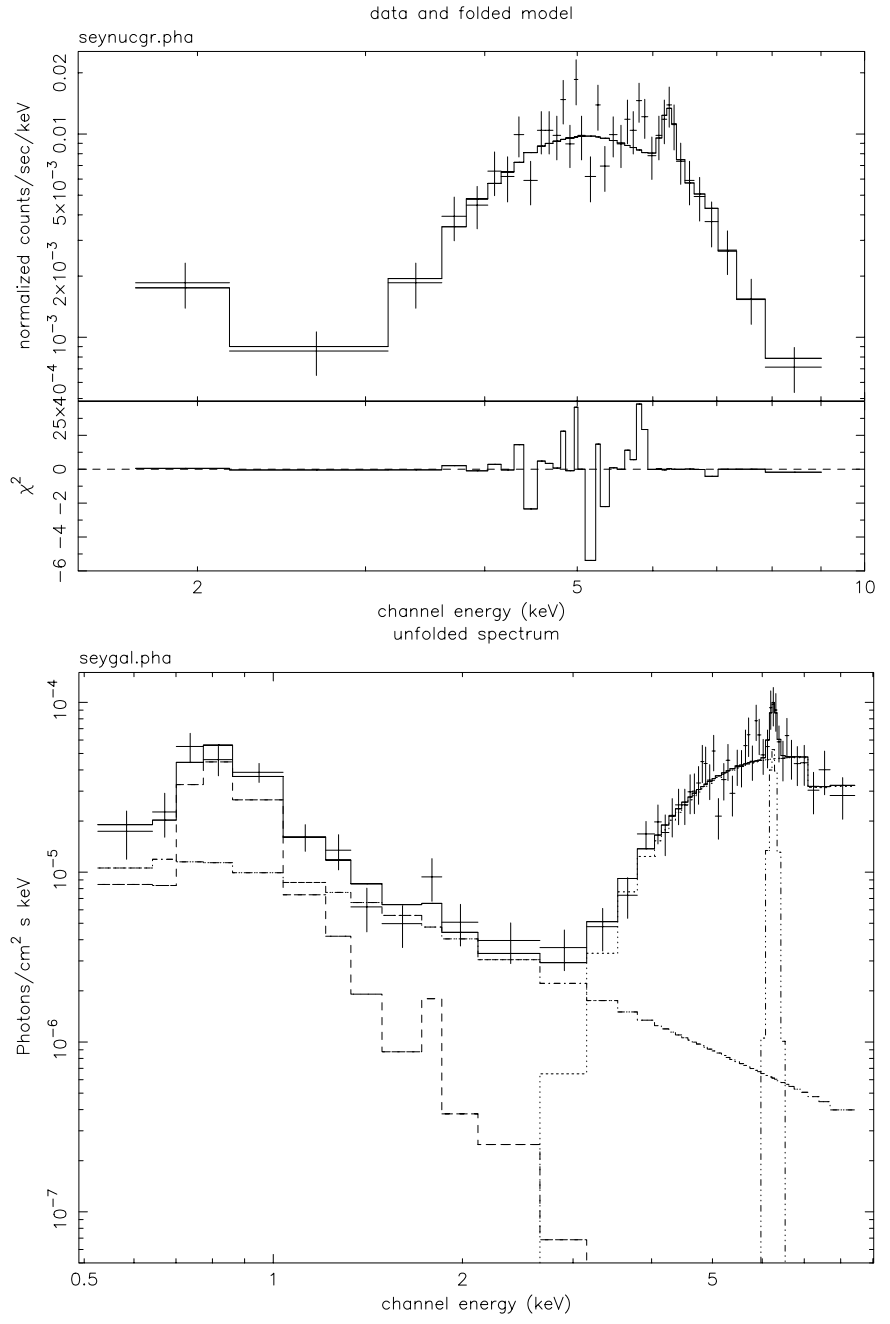

Fig. 8. Energy distribution of the counts from the entire $10^{\prime \prime}$ radius region centered on NGC 7319 (and $\chi^{2}$ contributions) are shown in the top panel. The unfolded spectrum and the three components used in the model (MEKAL with $k T \sim 0.65 \mathrm{keV}$; unabsorbed power law + absorbed power law with $N_{\mathrm{H}}$ (intrinsic) $\sim 4 \times 10^{23} \mathrm{~cm}^{-2}$ and $\Gamma \sim 1.7$; redshifted $6.4 \mathrm{keV}$ Gaussian line; see text) are shown in the bottom panel.

an intrinsic $L_{\mathrm{X}}(2-10 \mathrm{keV})=7 \times 10^{42} \mathrm{erg} \mathrm{s}^{-1}$. The high energy component from NGC 7319 was already discovered by ASCA (Awaki et al. 1997). The lower spatial resolution of that observation did not allow the authors to properly separate the Sey 2 contribution from more extended emission although all hard emission was assumed to be from the nucleus. The spectral fit was similar to the one derived here except that we find a significantly smaller equivalent width.

\section{Unraveling the complex X-ray morphology of $S Q$}

A key element in compact group evolutionary history involves stripping events. The distribution of neutral gas in SQ is a spectacular example of these processes that must involve multiple interactions with group members and/or intruders acquired from the surroundings. Studies of the events and conditions in SQ can therefore give insights into processes that are common in such galaxy aggregates. 


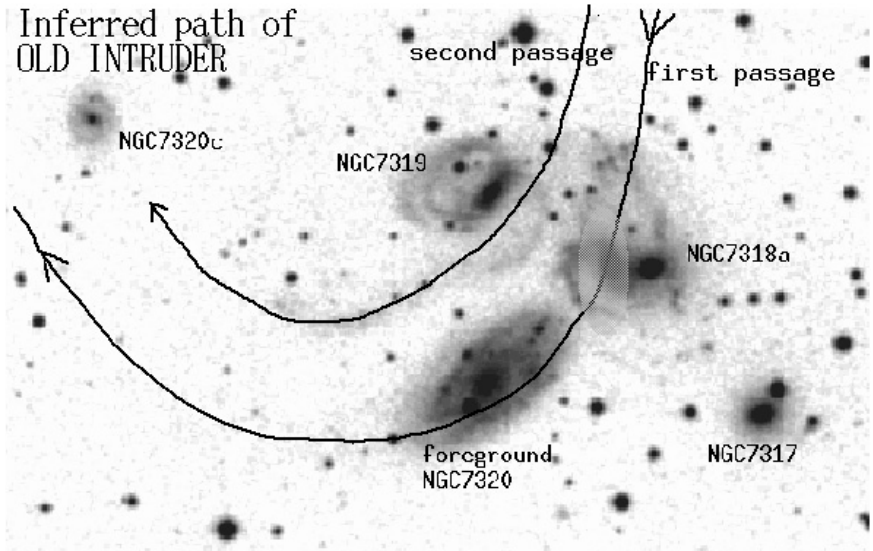

Fig. 9. A very schematic view of the presumed path of the old intruder NGC 7320c. The image of new intruder NGC 7318b is suppressed (shaded out) since it was not in SQ at the time.

Moles et al. (1997) proposed a dynamical model for SQ to explain the observational evidence. Using a larger body of multiwavelength data, S01 recently evaluated and tested their "two intruder" scenario, that interprets twin tidal tails as the products of two subsequent passages of NGC 7320c, the old intruder, through the group (cf. Fig. 9). The new intruder, NGC 7318b, is now entering SQ with a relative line of sight velocity of $\sim 1000 \mathrm{~km} \mathrm{~s}^{-1}$. Some aspects of the two intruder scenario are not shared by all investigators (e.g. Williams et al. 2002), but there is a consensus about the evidence of past and present episodes of interactions in SQ, that allow us to interpret much of the X-ray evidence in a self-consistent way.

The increased sensitivity of Chandra allows us to identify several components of extended X-ray emission. 1) The NS feature, a clumpy structure elongated in the NS direction (with possible branches towards NGC 7319 and the NW) closely coincident with the strongest radio continuum and optical line emission. 2) An irregular low surface brightness component surrounding the NS feature (radius $=1.5$ ). 3) Smaller scale extended emission coincident with NGC 7319 and NGC 7318a. Component 1) is almost certainly shock related while component 2) could involve shock and/or underlying diffuse emission. The most straightforward interpretation for components 3 ) involves an association with the respective galaxies. We cannot rule out a connection with the recent/ongoing collision because both galaxies, especially NGC 7318a, may lie in the path of the new intruder. Individual galaxies are discussed in Sect. 4.

\subsection{The shock zone in $S Q$}

The NS feature was previously interpreted as evidence for a large scale shock (Pietsch et al. 1997; S01). Chandra data reinforce this view with better evidence for (see Fig. 10): a) spatial coincidence between the NS feature and similar radio continuum, $\mathrm{H} \alpha$ and [ $\mathrm{N}_{\text {I I }}$ ] emission structures; b) lack of coincidence between the X-ray emission and the galaxies or extended stellar halo in SQ (Moles et al. 1998) and c) lack of X-ray coincidence with $\mathrm{HI}$ line emission in the velocity range of the new intruder NGC 7318b (S01). Optical spectroscopy (Xu et al. 2001) also

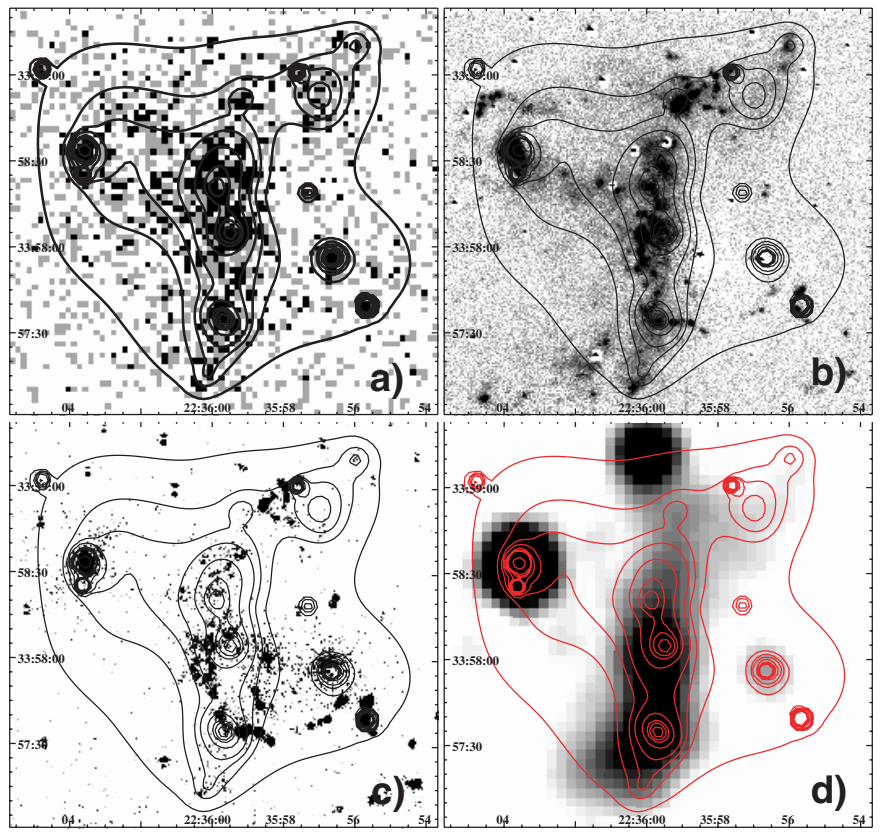

Fig. 10. X-ray contours (from the $0.5-2.0 \mathrm{keV}$ band adaptively smoothed map) superimposed on optical emission line and radio continuum data. Contours are overlayed on: a) $\mathrm{X}$-ray image, binned to $2^{\prime \prime} \times 2^{\prime \prime}$ pixels; b) $\mathrm{H} \alpha$ emission in the $6500 \mathrm{~km} \mathrm{~s}^{-1}$ SQ velocity range; c) $\mathrm{H} \alpha$ emission in the $5700 \mathrm{~km} \mathrm{~s}^{-1}$ new intruder velocity range; d) $1400 \mathrm{MHz}$ radio continuum emission. Images have all the same scale.

shows that emission line ratios from gas coincident with the NS region imply shock conditions. In the simplest scenario, the shock results from the collision of NGC $7318 \mathrm{~b}$ with previously stripped gas in SQ.

In the strong shock approximation, as expected from an impact with $v \sim 1000 \mathrm{~km} \mathrm{~s}^{-1}$, the shock temperature

$$
\begin{aligned}
T_{\mathrm{sh}} & =\frac{3}{16}\left(\mu \bar{m} / k_{\mathrm{B}}\right) \Delta v^{2} \sin ^{2} \phi \\
& \approx 2.7 \times 10^{7} \mu v_{8}^{2} \sin ^{2} \phi \quad \mathrm{K} .
\end{aligned}
$$

The quantities $\mu, \bar{m}, k_{\mathrm{B}}$, and $v_{8}$ are the mean molecular weight, mean particle mass of the gas, Boltzmann's constant, upstream velocity in units of $10^{8} \mathrm{~cm} \mathrm{~s}^{-1}$, respectively. In a perpendicular shock, the angle between the incoming flow direction and the shock surface $\phi=90^{\circ}$. We further assume $\mu=1$, which accounts for a completely neutral upstream gas and a perfect gas with ratio of specific heats, $\gamma_{c}=5 / 3$.

This shock temperature is significantly higher than that derived in Sect. 2.3 and cannot be interpreted as the result of significant cooling if the collision is ongoing (or even recent, see later). It is more likely that the shock conditions are not as simple as assumed in Eq. (1), and require either an oblique (small $\phi$, cf. Appendix A.1) and/or weak shock (i.e. the upstream medium is hot and has a sizeable counter-pressure) in which case the strong shock assumption breaks down. The effects of a magnetic field are also considered in Appendix A.2, but the field strength derived appears to be rather high.

If the new intruder collides with a neutral hydrogen cloud, as suggested by the spatial "continuity" between the NS feature and $\mathrm{H}_{\mathrm{I}}$ clouds $\mathrm{N}$ and $\mathrm{S}$ of it with consistent velocities (see 
Fig. 11), we expect strong shock conditions to prevail. To reconcile expected and observed post-shock temperatures, the incoming flow should cross the shock at an angle of $\phi \approx 30^{\circ}$, for an upstream $\mathrm{H}_{\mathrm{I}}$ temperature of $100 \mathrm{~K}$ (see Appendix A.2, Eq. (A.3)). Since the upstream Mach number, $M_{\infty} \sim 930$, is very high, the opening angle of the Mach cone, and thus also of the bow shock, should indeed be fairly small. Therefore we do not expect a significant amount of hotter gas to exist (e.g. in the stagnation point region, where the temperature is at most a factor of 4.5 higher). Thus the shock conditions look fairly reasonable, and although they may look somewhat specific, they are easily realized.

Due to the high Mach number, the compression ratio should be close to that for a perpendicular shock, e.g. $n_{\mathrm{X}} / n_{1} \approx 4$ (Eq. (A.1)). To evaluate the gas densities $n_{\mathrm{X}}$, we can assume the simplified spectral models derived in Sect. 2.3.1, that will give a good estimate of these quantities. In the NS feature, with a luminosity $L_{\mathrm{X}} \sim 1.5 \times 10^{41} \mathrm{erg} \mathrm{s}^{-1}$ in an ellipsoidal volume with $V=\frac{4}{3} \pi a b c \approx \frac{4}{3} \pi 11^{\prime \prime} \times 38^{\prime \prime} \times 11^{\prime \prime}(a, b, c$ denote the semi-major axes), the density:

$n_{\mathrm{X}}=\left(\frac{L_{\mathrm{X}}}{V_{\mathrm{X}} \Lambda\left(T_{\mathrm{X}}\right)}\right)^{1 / 2} \sim 2.7 \times 10^{-2} \mathrm{~cm}^{-3}$,

for $\Lambda\left(T_{\mathrm{X}}\right) \approx 7 \times 10^{-24}$, appropriate for gas with $10 \%$ solar metallicity (e.g. Böhringer \& Hensler 1989). This suggests a gas mass $M_{\text {gas }} \sim 6.5 \times 10^{8} M_{\odot}$, and a cooling time

$t_{\text {cool }} \simeq \frac{3 k_{\mathrm{B}} T_{\mathrm{X}}}{n_{\mathrm{X}} \Lambda\left(T_{\mathrm{X}}\right)} \approx 4.2 \times 10^{8} \mathrm{yr}$.

The actual density is likely to be locally higher (factors of $\sim 2$ ), since the emission is obviously clumpy and more compressed towards the W. In the low surface brightness region surrounding it, the average density is a factor $\sim 10$ lower, and at roughly the same temperature as the NS-feature, giving a cooling time also $\sim 10$ times longer. Thus the shock temperature could be explained by cooling only if the shock is $\gg 10^{8}$ year old which is inconsistent with other (all shorter) timescales (S01).

We can infer the pre-shock density $n_{\infty} \sim 6.5 \times 10^{-3} \mathrm{~cm}^{-3}$ as a mean value of $n_{1} \sim 3.4 \times 10^{-3}-1 \times 10^{-2} \mathrm{~cm}^{-3}$ derived for the $\mathrm{H}_{\mathrm{I}}$ densities in Arc-S and NW-HV clouds (from the data of Williams et al. 2002). We therefore derive a compression ratio $n_{\mathrm{X}} / n_{1} \approx 4$, consistent to the expected value (Eq. (A.1)).

\subsection{Constraints from UV, optical and radio continuum emission}

The large multiwavelength database available for SQ motivates additional considerations:

- The gas involved in the NS X-ray/H $\alpha+[\mathrm{NII}] 6583 /$ radio continuum feature, and at least some of the $\mathrm{H}_{\mathrm{I}}$ clouds, is a product of past tidal interactions. Ram pressure stripping is not very efficient, given the low densities involved and the low velocity dispersion in SQ. This suggest that NGC 7318b can retain a significant residual ISM after the encounter with SQ: $n_{\mathrm{IGM}} v_{\mathrm{N} 7381 \mathrm{~b}}^{2} \geq 2 n_{\mathrm{N} 7381 \mathrm{~b}} \sigma_{\mathrm{N} 7381 \mathrm{~b}}^{2}$ (Takeda et al. 1984) gives $\sigma_{\mathrm{N} 7381 \mathrm{~b}} \leq 100 \mathrm{~km} \mathrm{~s}^{-1}$, using $n_{\mathrm{IGM}} \sim 10^{-2} \mathrm{~cm}^{-3}$ for the IGM number density and $n_{\mathrm{N} 7381 \mathrm{~b}} \sim 1 \mathrm{~cm}^{-3}$ for the average galaxy's

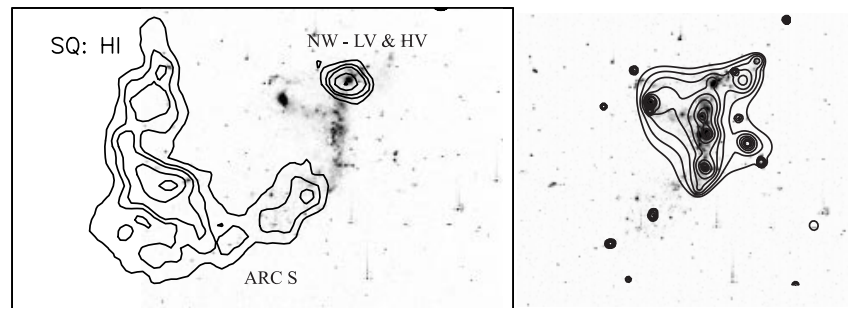

Fig. 11. Comparison of the spatial distribution of the neutral hydrogen (left; from Fig. 5 in S01) and X-rays (right) relative to $\mathrm{H} \alpha$ in the SQ velocity range. The $X$-ray panel covers an area of $\sim 5^{\prime} \times 4^{\prime}$.

ISM density. The derived velocity dispersion $\sigma_{\mathrm{N} 7381 \mathrm{~b}}$ corresponds to a galaxy mass of less than $\sim 3 \times 10^{10} M_{\odot}$.

- The NS feature is spatially (anti)correlated with the residual $\mathrm{H}$ I gas $\mathrm{N}$ and $\mathrm{S}$ of it. A lot of this gas may be related to the last passage of NGC 7320c through SQ. The central part of it has recently been shocked by NGC 7318b. The transverse velocity component is unknown and the direction of motion of NGC 7318b is therefore uncertain. Striations in the HST and CFHT images (S01) suggest motion from SW to NE or vice versa. Contour lines of the X-ray emission in NS feature suggest a higher compression on the $\mathrm{W}$ side, favouring motion from NE to SW.

- The low surface brightness emission surrounding the sharper NS feature could be interpreted as a preexisting hot IGM. The low velocity dispersion in SQ members requires it to have been heated by previous collisions (e.g. NGC 7320c?). The inferred trajectory in Fig. 9 and a projected distance of $\sim 120 \mathrm{kpc}$, allow us to estimate the projected velocity $\left\langle v_{O I}\right\rangle \sim \frac{120 \mathrm{kpc}}{3 \times 10^{8} \mathrm{yr}} \approx$ $400 \mathrm{~km} \mathrm{~s}^{-1}$, which is a lower limit. According to Eq. (1) this velocity corresponds to $T \sim 6 \times 10^{6} \mathrm{~K}$, in excellent agreement with the measured X-ray temperature. The long cooling time derived above relative to the inferred last passage of NGC 7320c suggests that such a hot IGM component would not have cooled significantly over this period. The rather uniform distribution of the low surface brightness emission (in contrast to e.g. the NS feature) would be due to the equilibration by shock and/or sound waves that were able to propagate through the IGM within at least the last $3 \times 10^{8} \mathrm{yr}$ (sound crossing time scale). We expect that the impact of NGC $7318 \mathrm{~b}$ will produce only a weak shock here, given the relatively high gas temperature. If this component is related to the ISM of NGC 7318b, rather than residual hot gas in SQ, we must assume that it has expanded and cooled from an expected postshock temperature given by Eq. (1).

- The X-ray/radio continuum/optical emission line extension or tail towards the NW is a puzzle if the collision is ongoing. The X-ray cooling timescale is about an order of magnitude longer than the new intruder crossing $\left(t_{\mathrm{c}}(\mathrm{NGC} 7318 \mathrm{~b}) \sim\right.$ few $X$ $10^{7}$ years $)$. If the former timescale were a better estimate for the age of the shock we could more easily account for the NW extension as a tidal feature.

- The X-ray gap shown in Fig. 5 is an additional puzzle although similar features have been observed in richer environments. The prominent "cold front" observed in the cluster A3776 is interpreted as colder gas moving into a hotter ambient 
medium (Vikhlinin et al. 2001). Mazzotta et al. (2002) identify two additional features, one of which is an arc-like filamentary $\mathrm{X}$-ray depression oriented perpendicular to the direction of motion. Large scale hydrodynamic instabilities coupled with magnetic field effects are suggested to explain these morphological peculiarities. The surface brightness discontinuity seen in SQ appears sharper and more prominent than in the other examples. A temperature discontinuity is associated with the surface brightness edges in other examples and we cannot detect such a feature in SQ. On the other hand, the temperatures of both the ambient gas and the NS shock feature in SQ are significantly lower than the gas in A3667. This could cause a more significant drop in emissivity if the temperature falls below the energies at which the instruments are sensitive. Forthcoming observations with XMM-Newton, that will give more spectral information, will hopefully provide clues to its nature.

\section{Discrete sources in the $S Q$ galaxies}

A few discrete sources can be identified with galaxies in the group.

NGC 7319: NGC 7319 is dominated by a strong heavily absorbed nuclear point source embedded in fainter extended emission that extends to $\sim 10^{\prime \prime}$ radius. Three additional sources $(\# 9,10,13)$ are associated with this galaxy: source \#9 is near an optical point source and the other two are projected on the spiral arms but do not appear to coincide with individual sources or with specific condensations (see Fig. 12).

A connection between interactions and AGN activity has been difficult to prove conclusively although recent evidence has been advanced for an excess of Seyfert 2 nuclei in a reasonably complete sample of compact groups (Coziol et al. 2000). NGC 7319 shows both evidence for a past interaction event that stripped it of its ISM (tied to the last passage of NGC 7320c and the generation of the younger optical tidal tail) and near nuclear activity in the form of an emission line jet (Aoki et al. 1996) and triple lobed radio (Aoki et al. 1999) structure on a 10" scale that cannot be so easily related to past interaction episodes. One can argue that the quasi-continuous nature of the tidal perturbations in compact groups might more efficiently channel gas into nuclei giving rise to phenomena of this kind. Interpretation of the extended X-ray emission around NGC 7319 is complicated by the presence of this optical jet and also by the nearby unresolved X-ray source (source \#9). The most straightforward assumption is that source \#9 is unrelated to the nuclear activity. That assumption may be challenged by the discovery of other AGN with nearby unresolved X-ray sources (Mrk 3, Morse et al. 1995; NGC 4258, Wilson et al. 2001a): in NGC 4151 (Yang et al. 2001) and Pictor A (Wilson et al. 2001b). Source \#9 is $\sim 8^{\prime \prime} \mathrm{S}$ from the nucleus (Fig. 12) and may coincide with a compact optical object (\#54 in Gallagher et al. 2001). Its BVI colors are consistent with a late $F$ to early $G$ main sequence star but the observed magnitude would put it at $\sim 30 \mathrm{kpc}$ without taking into account any possible extinction. It is therefore unlikely to be a galactic star. It is embedded in a region where $\mathrm{CO}$ is detected (Yun et al. 1997) which indicates that star formation may be occurring there.

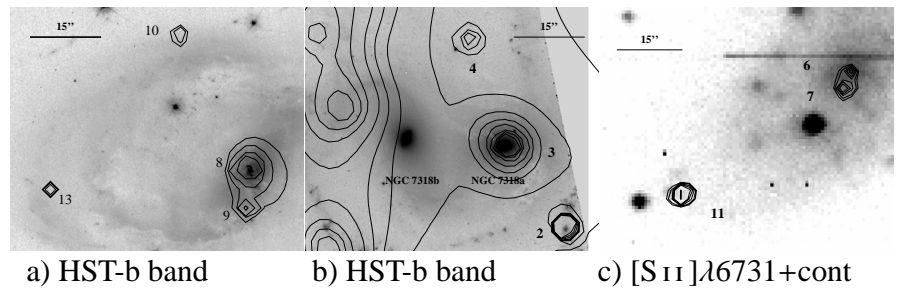

a) HST-b band

b) HST-b band

c) $\left[\mathrm{S}_{\text {I I }}\right] \lambda 6731+\mathrm{cont}$

Fig. 12. Close-up of several interesting regions in SQ. The X-ray contours are superimposed on an HST WFPC2 $B$-band image in panel a) and b) and a [S I $] \lambda 6731$ + continuum image showing NGC 7320 in panel c). The scale of $15^{\prime \prime}$ is given in each panel. Source numbers from Table 1.

Given the high X-ray luminosity of X-ray point sources assumed to belong to NGC $7319, L_{X} \geq 5 \times 10^{39} \mathrm{erg} \mathrm{s}^{-1}$, they may be analogs of the Ultra Luminous X-ray sources (ULX), that are found in actively star forming galaxies (Zezas et al. 1999; Roberts \& Warwick 2000; Fabbiano et al. 2001) and some in more normal spiral galaxies (Tennant et al. 2001; Prestwich 2002). A definite interpretation about the origin of ULX is not yet available. Both spectral characteristics and variability arguments suggest that they are binary accretion sources, although the requirement of a sizeable central collapsed object might favour beaming or anisotropy in the accretion process (King et al. 2001; Körding et al. 2002). Sources 10 and 13 are in a region of NGC 7319 with colors (S01) consistent with a star formation event that ended about the time it was stripped of its ISM (a few times $10^{8}$ years ago).

NGC 7318a. A relatively hard source is coincident with the nucleus, but a broad band $(0.5-5.0 \mathrm{keV})$ radial profile is inconsistent with the Chandra Point Spread Function, suggesting that the emission is extended and likely related to the entire galaxy. This elliptical galaxy appears to lie directly in the path of the ongoing collision. It is the only galaxy in SQ other than NGC 7319 that shows radio emission. All Hi and Hi that are found $\mathrm{N}, \mathrm{W}$ and $\mathrm{E}$ of this galaxy belong to new intruder NGC 7318b. One can therefore infer an "inverse-Cartwheel" scenario where the disk of NGC $7318 \mathrm{~b}$ has passed directly through this galaxy. While it is tempting to say "yes" to such an hypothesis, two arguments warrant caution: 1) the X-ray (and radio) properties of NGC 7318a are not unlike more isolated galaxies of the same type and 2) the high velocity of the new intruder leaves little time for the formation of interaction induced structure or activity, unless the collision is "old", as discussed earlier. Of course in situ shocking of gas within the galaxy is not subject to the latter objection.

NGC 7317, NGC 7320c, NGC 7318b. None of these galaxies are formally detected with Chandra. An enhancement is visible at the position of NGC 7317. We measure $11 \pm 4$ counts in the $0.3-10 \mathrm{keV}$ band within an area with $\sim 4$ " radius, which corresponds to an unabsorbed flux $f_{\mathrm{X}} \sim 5 \times 10^{-15} \mathrm{erg} \mathrm{cm}^{-2} \mathrm{~s}^{-1}$. This value can be used as an upper limit for X-ray emission from the other two galaxies. This is particularly true for NGC $7318 \mathrm{~b}$ where complex emission from the NS feature precludes a direct measure.

Isolated bright sources. X-ray sources \#2, 5, and possibly 4, are detected close to and possibly associated with bright emission 
regions SW and N of NGC 7318ab. All such emission regions show velocities consistent with the "b" galaxy (S01). Source \#5 could be associated with "putative star" \#127 in Gallagher et al. (2001). An enhancement is also seen coincident with ISO detected starburst A (Xu et al. 1999) this time with an SQ velocity. The inferred luminosities are relatively high and consistent with ULX sources in starforming regions.

NGC 7320 We find two sources (\#6 and 7) in the nuclear region of NGC 7320 with luminosities $\sim 6$ and $\sim 10 \times 10^{37} \mathrm{erg} \mathrm{s}^{-1}$. Source \#6 appears to coincide with the nucleus (see Fig. 12) and would represent a relatively low luminosity nuclear source comparable to a bright binary system. A third source, \#11 at the SE edge of NGC 7320 shows a luminosity of $\sim 2 \times 10^{38} \mathrm{erg} \mathrm{s}^{-1}$. Both extranuclear sources are most likely bright accreting binary systems similar to the ones found in other spiral galaxies. A more speculative interpretation would relate source 11 to the old tidal tail passing behind NGC 7320, in which case $L_{\mathrm{X}}$ would exceed $\sim 10^{39} \mathrm{erg} \mathrm{s}^{-1}$ at the distance of SQ.

\section{Summary and conclusions}

New high sensitivity and high resolution Chandra observations confirm the complexity of the X-ray emission in SQ. The most prominent sources are associated with a large scale shock that is strongest at low energies $(E<1.5 \mathrm{keV})$ and a Seyfert 2 nucleus in NGC 7319 that dominates the emission above $2 \mathrm{keV}$. Additional sources are likely associated with members of SQ and with the foreground galaxy NGC 7320. Low surface brightness diffuse emission is also detected in the core of the system associated either with a large scale IGM or with the shocked ISM of the new intruder NGC 7318b.

The complex dynamical history of SQ offers the most plausible explanation for the large scale NS shock as a collision between a high velocity intruder and a gaseous debris field produced by earlier interacting events. Analytical evaluations of the shock conditions, taking into account the X-ray morphology and spectrum, require an oblique shock propagating into a pre-existing $\mathrm{H}_{\mathrm{I}}$ cloud. The alternative is to postulate that the collision is not ongoing and that the shock has cooled considerably.

Detailed X-ray analysis of compact groups can also provide us with further insights into the problem of IGM metal enrichment and, in particular, whether galaxy interactions rather than galactic winds are the primary process for entropy and heavy element input into the IGM. The spectral fits presented here are carried out under the assumption of collisional ionization equilibrium. They suggest a low IGM metallicity and a preponderance of tidal interactions over galactic outflows. More detailed investigations are not warranted by the statistics of the Chandra data. Analysis of new XMM-Newton data will provide more stringent constraints on spectral and dynamical properties of the collision scenario discussed here. Stephan's Quintet continues to be an excellent laboratory for studying dynamics and evolution in compact groups and represents one of the most useful local analogs of phenomena thought to be much more common at high redshift.

Acknowledgements. G.T. thanks G. Hasinger, J. Trümper and all colleagues at the Max-Planck-Institut für extraterrestrische Physik (MPE) for fruitful discussions and hospitality while part of this work was done. GT acknowledges support from grants from the Italian Space Agency (ASI). D.B. thanks G. Hasinger and the MPE for financial support. J.S. acknowledges financial support under NASA grant GO0$1142 X$.

The compressed files of the "Palomar Observatory - Space Telescope Science Institute Digital Sky Survey" of the northern sky, based on scans of the Second Palomar Sky Survey are copyright (c) 1993-2000 by the California Institute of Technology and are distributed by agreement. All Rights Reserved.

\section{Appendix A: Shock conditions for interaction of new intruder with IGM}

General shock physics tells us that a supersonically moving body will have a bow shock at its leading edge. In the rest frame of the high velocity intruder the incoming flow will enter the shock wave at some angle $\phi$, allowing the downstream material to be deflected and flow subsonically around the galaxy (e.g. the NW star forming region).

It is known from aerodynamics that the shape of the bow shock and its stand-off distance (gap between the nose of the obstacle and the bow shock) cannot be determined from hydrodynamics alone. It also depends on the geometry since the fluid equations do not exhibit any characteristic length scale (for a perfect gas). The bow shock can be viewed as a transition from a planar/perpendicular (near the stagnation point region) to an oblique shock. In the following, we will quantitatively analyze these two situations. In the planar case, in which the maximum compression occurs, a magnetic field, parallel to the shock surface for the maximum effect, will also be considered. In the next subsections we discuss simple analytic expressions that we have used for the most plausible scenarios. We will use the subscripts "sh" and " $\infty$ " for downstream and upstream quantities, respectively, and an adiabatic gas with ratio of specific heats $\gamma_{c}=5 / 3$. $M$ denotes the Mach number, $c$ the speed of sound, and $v_{A, \infty}=\sqrt{B /(4 \pi \rho)}$ the Alfvén velocity.

\section{A.1. Oblique shock}

The compression and the temperature ratio for an oblique shock associated with a bow shock will be

$$
\begin{aligned}
\frac{\rho_{\phi}}{\rho_{\infty}}= & \frac{(\gamma+1) M_{\infty}^{2} \sin ^{2} \phi}{(\gamma-1) M_{\infty}^{2} \sin ^{2} \phi+2} \approx 4, \\
\frac{T_{\phi}}{T_{\infty}}= & \frac{\left[(\gamma-1) M_{\infty}^{2} \sin ^{2} \phi+2\right]}{(\gamma+1)^{2} M_{\infty}^{2} \sin ^{2} \phi} \\
& \times \frac{\left[2 \gamma M_{\infty}^{2} \sin ^{2} \phi-(\gamma-1)\right]}{(\gamma+1)^{2} M_{\infty}^{2} \sin ^{2} \phi} \\
\approx & \frac{2 \gamma(\gamma-1)}{(\gamma+1)^{2}} M_{\infty}^{2} \sin ^{2} \phi \\
= & \frac{5}{16} M_{\infty}^{2} \sin ^{2} \phi .
\end{aligned}
$$

Note that Eq. (A.2) is the generalization of Eq. (1) for arbitrary shock strengths. A detailed numerical calculation, which is beyond the scope of this paper, will be necessary for a more quantitative comparison, and it should include a temperature structure downstream of the bow shock. 


\section{A.2. Perpendicular MHD shock}

Using the above notation, we can write the compression ratio $r$ and the ratio of gas to magnetic pressure in the upstream plasma $\beta_{\infty}$ as

$r=\frac{\rho_{\mathrm{sh}}}{\rho_{\infty}}=\frac{v_{\infty}}{v_{\mathrm{sh}}}=\frac{B_{\mathrm{sh}}}{B_{\infty}}$,

$\beta_{\infty}=\frac{2 c_{\infty}^{2}}{\gamma v_{A, \infty}^{2}}=\frac{8 \pi \rho_{\infty} k_{\mathrm{B}} T_{\infty}}{B_{\infty}^{2} \bar{m}}$,

and from the Rankine-Hugoniot conditions we can derive the following equations:

$$
\begin{aligned}
2(2- & \gamma) r^{2}-\gamma(1+\gamma) \beta_{\infty} M_{\infty}^{2} \\
& +\gamma r\left\{2+\beta_{\infty}\left[2+(\gamma-1) M_{\infty}^{2}\right]\right\}=0,
\end{aligned}
$$

and

$\frac{T_{\mathrm{sh}}}{T_{\infty}}=\frac{1}{r}\left[1+\frac{1-r^{2}}{\beta_{\infty}}+\gamma\left(1-\frac{1}{r}\right) M_{\infty}^{2}\right] ;$

for $\beta_{\infty} \rightarrow \infty$ we recover the ordinary gas shock relations (see Landau \& Lifshitz 1959). Equation (A.7) can be used to derive the magnetic field strength in the uncompressed gas due to

$B_{\infty}=\sqrt{\frac{8 \pi k_{\mathrm{B}} n_{\infty} T_{\infty}}{\left(1-r^{2}\right)}\left[r \frac{T_{\mathrm{sh}}}{T_{\infty}}-1-\gamma M_{\infty}^{2}\left(1-\frac{1}{r}\right)\right]}$.

For the shock propagating into the NS feature to be compressive it has to be a fast MHD shock, satisfying the condition: $v_{\mathrm{N} 7318 \mathrm{~b}} \geq c_{\infty}^{2}+v_{A, \infty}^{2}$.

If the pre-shock material is the "missing" part of an $\mathrm{H}_{\mathrm{I}}$ structure connecting the Arc-S and NW-HV regions in Williams et al. (2002, see Fig. 11), its inferred density $n_{\infty} \sim$ $6.5 \times 10^{-3} \mathrm{~cm}^{-3}$ and upstream temperature $T_{\infty}=100 \mathrm{~K}$. Thus $c_{\infty} \approx 1.1 \mathrm{~km} \mathrm{~s}^{-1}$ and the shock is indeed hypersonic since the Mach number is $M_{\infty} \approx 932$. In case of a strong shock $\left(M_{\infty} \gg 1\right)$ Eq. (A.6) yields a compression ratio of $r=4$ for the derived X-ray quantities. This results in an unshocked magnetic field strength of $B_{\infty} \approx 1.13 \times 10^{-5} \mathrm{G}$ and thus, in the strong shock approximation, of a factor 4 higher in the shocked NS feature. As the Alfvén speed equals $v_{A, \infty} \approx 280 \mathrm{~km} \mathrm{~s}^{-1}$, the fast MHD shock condition is easily fulfilled. Since $B_{\infty} \propto 1 / r$ the magnetic field strength decreases with the compression ratio $r$; therefore very low compression ratios are disfavored in a perpendicular MHD shock case. Even the field value for $r=4$ seems to be on the high side.

\section{References}

Aoki, K., Ohtani, H., Yoshida, M., \& Kosugi, G. 1996, AJ, 111, 140 Aoki, K., Kosugi, G., Wilson, A. S., \& Yoshida, M. 1999, ApJ, 521, 565

Arp, H., \& Lorre, J. 1976, ApJ, 210, 58

Awaki, H., Koyama, K., Matsumoto, H., et al. 1997, PASJ, 49, 445

Böhringher, H., \& Hensler, G. 1989, A\&A, 215, 147
Breitschwerdt, D., \& Schmutzler, T. 1999, A\&A, 347, 650

Coziol, R., Iovino, A., \& de Carvalho, R. R. 2000, AJ, 120, 47

Della Ceca, R., Castelli, G., Braito, V., Cagnoni, I., \& Maccacaro, T. 1999, ApJ, 524, 674

Fabbiano, G., Zezas, A., \& Murray, S. S. 2001, ApJ, 554, 1035

Gallagher, S. C., Charlton, J. C., Hunsberg, S. D., Zaritsky, D., \& Whitmore, B. C. 2001, AJ, 122, 163

Hickson, P. 1982, ApJ, 255, 382

Hickson, P., \& Rood, H. 1988, ApJ, 331, L69

Hicks, A., Wise, M. W., Houck, J., \& Canizares, C. R. 2002, ApJ, 580, 763

King, A. R., Davies, M. B., Ward, M. J., Fabbiano, G., \& Elvis, M. 2001, ApJ, 552, L109

Körding, E., Falcke, H., \& Markoff, S. 2002, A\&A, 383, 854

Landau, L. D., \& Lifshitz, E. M. 1959, Course of theoretical physics (Oxford: Pergamon Press)

Makishima, K., Kubota, A., Mizuno, T., et al. 2000, ApJ, 535, 632

Mazzotta, P., Fusco-Femiano, R., \& Vikhlinin, A. 2002, ApJ, 549, L31

Mendes de Oliveira, C., Plana, H., Amram, P., Balkowski, C., \& Bolte, M. 2001, AJ, 1212524

Moles, M., Sulentic, J. W., \& Marquez, I. 1997, ApJ, 485, L69

Moles, M., Marquez, I., \& Sulentic, J. W. 1998, A\&A, 334, 473

Moran, E. C., Kay, L. E., Davis, M., Filippenko, A. V., \& Barth, A. J. 2001, ApJ, 556, 75

Morse, J. A., Wilson, A. S., Elvis, M., \& Weaver, K. A. 1995, ApJ, 439,121

Pietsch, W., Trinchieri, G., Arp, H., \& Sulentic, J. W. 1997, A\&A, 322,89

Plana, H., Mendes de Oliveira, C., Amram, P., et al. 1999, ApJ, 516, 69

Prestwich, A. H. 2002, in High-Energy Universe at Sharp Focus: A Symposium of Chandra Science, ed. E. M. Schlegel \& S. Vrtilek, ASP Conf. Ser., in press

Raymond, J. C., \& Smith, B. W. 1997, ApJS, 35, 419

Roberts, T. P., \& Warwick, R. S. 2000, MNRAS, 31598

Sulentic, J., \& de Mello Rabaca, D. 1993, ApJ, 410, 520

Sulentic, J. W., Rosado, M., Dultzin-Hacyan, et al. 2001, AJ, 122, 2993 (S01)

Takeda, H., Nulsen, P. E. J., \& Fabian, A. C. 1984, MNRAS, 208, 261

Tennant, A. F., Wu, K., Ghosh, K. K., Kolodziejczak, J. J., \& Swartz, D. A. 2001, ApJ, 549, 43

Trinchieri, G., \& Goudfrooij, P. 2002, A\&A, 386, 472

Verdes-Montenegro, L., Yun, M. S., Williams, B. A., et al. 2001, A\&A, 377812

Vilchez, J., \& Iglesias-Paramo, L. 1998, ApJ, 506, L101

Vikhlinin, A., Markevitch, \& Murray, S. S. 2001, ApJ, 551, 160

Williams, B. A., Yun Min, S., \& Verdes-Montenegro, L. 2002, AJ, 123,2471

Wilms, J., Reynolds, C. S., Begelman, M. C., et al. 2001, MNRAS, 328,27

Wilson, A. S., Yang, Y., \& Cecil, G. 2001a, ApJ, 560, 689

Wilson, A. S., Yang, Y., \& Shopbell, P. L. 2001b, ApJ, 547, 740

Xu, C., Sulentic, J. W., \& Tuffs, R. 1999, ApJ, 512, 178

Xu, C., Gao, Y., Lu, N., \& Condon, J. J. 2001, in Gas and Galaxy Evolution, ed. J. Hibbard, M. Rupen, \& J. van Gorkom, ASP Conf. Ser., 240, 594

Yang, Y., Wilson, A. S., \& Ferruit, P. 2001, ApJ, 563, 124

Yun, M. S., Verdes-Montenegro, L., del Olmo, A., \& Perea, J. 1997, ApJ, 475, 21

Zezas, A. L., Georgantopoulos, I., \& Ward, M. J. 1999, MNRAS, 308, 302 